\title{
Adaptive Sliding Mode Control of MEMS Gyroscope Based on Neural Network Approximation
}

\author{
Yuzheng Yang and Juntao Fei \\ Jiangsu Key Laboratory of Power Transmission and Distribution Equipment Technology, College of IOT Engineering, \\ Hohai University, Changzhou 213022, China \\ Correspondence should be addressed to Juntao Fei; jtfei@yahoo.com
}

Received 21 December 2013; Revised 5 March 2014; Accepted 9 March 2014; Published 8 April 2014

Academic Editor: Huijun Gao

Copyright (C) 2014 Y. Yang and J. Fei. This is an open access article distributed under the Creative Commons Attribution License, which permits unrestricted use, distribution, and reproduction in any medium, provided the original work is properly cited.

\begin{abstract}
An adaptive sliding controller using radial basis function (RBF) network to approximate the unknown system dynamics microelectromechanical systems (MEMS) gyroscope sensor is proposed. Neural controller is proposed to approximate the unknown system model and sliding controller is employed to eliminate the approximation error and attenuate the model uncertainties and external disturbances. Online neural network (NN) weight tuning algorithms, including correction terms, are designed based on Lyapunov stability theory, which can guarantee bounded tracking errors as well as bounded NN weights. The tracking error bound can be made arbitrarily small by increasing a certain feedback gain. Numerical simulation for a MEMS angular velocity sensor is investigated to verify the effectiveness of the proposed adaptive neural control scheme and demonstrate the satisfactory tracking performance and robustness.
\end{abstract}

\section{Introduction}

MEMS gyroscopes have become the most growing microsensors for measuring angular velocity in recent years due to their compact size, low cost, and high sensitivity. Fabrication imperfections always result in some cross stiffness and damping effects and the performance of the MEMS gyroscope is deteriorated by the effects of time varying parameters, quadrature errors, and external disturbances. Therefore it is necessary to utilize advanced control methods to measure the angular velocity and minimize the cross coupling terms.

During the past few years, advanced control approaches have been proposed to control the MEMS gyroscope. Leland [1] presented an adaptive controller for tuning the natural frequency of the drive axis of a vibratory gyroscope. Park et al. [2] presented an adaptive controller for a MEMS gyroscope which drives both axes of vibration and controls the entire operation of the gyroscope. Batur et al. [3] developed a sliding mode controller and adaptive controller for a MEMS gyroscope. Robust adaptive controller has been developed using adaptive sliding mode control to control the vibration of MEMS gyroscope [4]. Tsai and Sue [5] proposed integrated model reference adaptive control and time-varying angular rate estimation algorithm for micromachined gyroscopes. Raman et al. [6] developed a closed-loop digitally controlled MEMS gyroscope using unconstrained sigma-delta force balanced feedback control. Since neural network has the capability to approximate any nonlinear function over the compact input space, RBF's properties make them attractive for interpolation and functional modeling. Therefore neural network's learning ability makes it a useful tool for adaptive application. Lewis et al. [7, 8] developed neural network approaches for robot manipulator. Feng [9] presented a compensating scheme for robot tracking based on neural networks and Lin and Chen [10] used neural network based robust nonlinear control for a magnetic levitation system. Park et al. [11] developed adaptive neural sliding mode control for the nonholonomic wheeled mobile robots with model uncertainty. Sadati and Ghadami [12] derived adaptive multimodel sliding mode control of robotic manipulators using soft computing. Lee and Choi [13] used neural network based robust nonlinear control for a magnetic levitation system. Lin et al. [14] developed an adaptive RBF neural network controller for robot manipulators. Huang et al. [15] developed a novel RBF sliding mode controller for a dynamic absorber. An adaptive neural compensation scheme 
without sliding mode method for tracking control of MEMS gyroscope was proposed [16]. In [17], robust adaptive sliding mode control is utilized to estimate the angular velocity of MEMS triaxial gyroscope, and neural network is adopted to estimate the upper bound of system nonlinearities. In [18], an input-output linearization technique is incorporated into the neural adaptive tracking control to cancel the nonlinearities and the adaptive neural network is used to perform the linearization control law. In this paper, different from [16-18], neural controller is proposed to approximate the unknown system model and sliding mode controller is employed to eliminate the approximation error and attenuate the model uncertainties and external disturbances. In order to improve the tracking performance of MEMS gyroscope, a robust adaptive control strategy of MEMS gyroscope using neural sliding compensator is developed. The proposed control strategy has the following advantages.

(1) An adaptive neural sliding mode control is adopted to on-line mimic the unknown model and compensate the approximation error. It incorporates RBF neural network into the adaptive sliding control system of MEMS gyroscope to learn the approximate model. Adaptive neural sliding mode compensation scheme is proposed to deal with the model approximation in the presence of model uncertainties and external disturbances.

(2) This paper integrates adaptive control, sliding mode control, and the nonlinear approximation of neural network control. Neural controller is proposed to approximate the unknown system model and sliding controller is employed to eliminate the approximation error and attenuate the model uncertainties and external disturbances.

(3) The proposed adaptive neural sliding controller does not need to establish accurate mathematical model and has the ability to approximate the nonlinear characteristics of system model. The proposed adaptive neural network sliding mode controller can guarantee the stability of the closed-loop system and improve the robustness for external disturbances and model uncertainties.

The paper is organized as follows. In Section 2, the dynamics of MEMS gyroscope is described. In Section 3, problem is formulated. In Section 4, an adaptive RBF network compensation is derived to guarantee the asymptotic stability of the closed-loop system. Simulation results are presented in Section 5 to verify the effectiveness of the proposed adaptive neural compensation control. Conclusions are provided in Section 6 .

\section{Dynamics of MEMS Gyroscope}

The dynamics of vibratory MEMS gyroscope is described in this section. A two-axis MEMS vibratory gyroscope mainly contains three parts: one is the sensitive element of single proof mass $(m)$ suspended by spring beams, another is electrostatic actuations and sensing mechanisms for forcing an oscillatory motion and sensing the position and velocity of the sensitive element, and the other is the rigid frame which is rotated along the rotation axis.

A schematic model of a two-axis MEMS vibratory gyroscope is shown in Figure 1. As an electromechanical system for special use, the initial and the most important step is to derive the motion equations. In this section the motion equations of MEMS gyroscope are derived based on Lagrange equation:

$$
\frac{\mathrm{d}}{\mathrm{d} t}\left(\frac{\partial L}{\partial \dot{x}_{i}}\right)-\frac{\partial L}{\partial x_{i}}+\frac{\partial F}{\partial \dot{x}_{i}}=Q_{i},
$$

where $L=E_{K}-E_{P}$ is Lagrange's function, $E_{K}$ and $E_{P}$ are kinetic and potential energies of the sensitive element, respectively, $F$ denote the generalized damping forces, $Q_{i}$ are generalized forces acting on the sensitive element, and $i$ ranges from 1 to the number of degrees of freedom under consideration. In our case it will be 2 .

Let us introduce the right-handed orthogonal reference frame $O X Y$ in which drive oscillation are excited along the $x$-axis and sense oscillation occurs along the $y$-axis. Assume a generalized coordinate $x$ as the displacement of the sensitive element along the $x$-axis. Similarly generalized coordinate $y$ corresponds to the displacements of the sensitive element along the $y$-axis. The arbitrary angular rate $\Omega$ is defined by its projections on the introduced above reference frame as $\Omega=\left\{\Omega_{x}, \Omega_{y}, \Omega_{z}\right\}$, where the third $z$-axis is perpendicular to the paper.

The total kinetic energy of the sensitive element in our case will be

$$
E_{K}=\frac{m}{2}\left[\left(\dot{x}-y \Omega_{z}\right)^{2}+\left(\dot{y}+x \Omega_{z}\right)^{2}+\left(y \Omega_{x}-x \Omega_{y}\right)^{2}\right] .
$$

Potential energy of the sensitive element is formed by stiffness of its springs and is given by

$$
E_{P}=\frac{k_{x}}{2} x^{2}+\frac{k_{y}}{2} y^{2},
$$

where $k_{x}$ is the total stiffness of the elastic suspension along the $x$-axis and $k_{y}$ is the total stiffness along the $y$-axis. Now using the Lagrange equation (1), the following motion equations that reveal the dynamics of MEMS gyroscope will appear after some simple transformations:

$$
\begin{aligned}
m \ddot{x}+ & d_{x x} \dot{x}+m\left(\omega_{x}^{2}-\Omega_{y}^{2}-\Omega_{z}^{2}\right) x \\
& \quad-2 m \Omega_{z} \dot{y}+m\left(\Omega_{x} \Omega_{y}+\dot{\Omega}_{z}\right) y=u_{x} \\
m \ddot{y}+ & d_{y y} \dot{y}+m\left(\omega_{y}^{2}-\Omega_{x}^{2}-\Omega_{z}^{2}\right) y \\
& +2 m \Omega_{z} \dot{x}+m\left(\Omega_{x} \Omega_{y}-\dot{\Omega}_{z}\right) x=u_{y},
\end{aligned}
$$

where $\omega_{x}^{2}=k_{1} / m$ and $\omega_{y}^{2}=k_{2} / m$ are natural frequencies of drive and sense oscillations mode, respectively. $d_{x x} \dot{x}$ and $d_{y y} \dot{y}$ are the damping forces terms. $-m\left(\Omega_{y}^{2}+\Omega_{z}^{2}\right) x$ and $-m\left(\Omega_{x}^{2}+\Omega_{z}^{2}\right) y$ are called centripetal forces. $m \dot{\Omega}_{z} y$ and $m \Omega_{z} \dot{x}$ are Euler forces. $2 m \Omega_{z} \dot{y}$ and $2 m \Omega_{z} \dot{x}$ are the Coriolis forces 
and used to reconstruct the unknown external angular rate $\Omega_{z} \cdot u_{x}$ and $u_{y}$ are external forces or control inputs.

Assuming that the external angular rate is almost constant over a long enough time interval, under typical assumptions $\Omega_{x} \approx \Omega_{y} \approx 0$, only the $z$-axis component of the angular rate $\Omega_{z}$ causes a dynamic coupling between the two axes. Taking fabrication imperfections into account, which cause extra coupling between the two axes, the motion equations of a MEMS gyroscope is finally given by

$$
\begin{aligned}
& m \ddot{x}+d_{x x} \dot{x}+d_{x y} \dot{y}+k_{x x} x+k_{x y} y=u_{x}+d_{x}+2 m \Omega_{z} \dot{y} \\
& m \ddot{y}+d_{x y} \dot{x}+d_{y y} \dot{y}+k_{x y} x+k_{y y} y=u_{y}+d_{y}-2 m \Omega_{z} \dot{x},
\end{aligned}
$$

where $d_{x x}, d_{y y}, k_{x x}$, and $k_{y y}$ are damping and spring coefficients; $d_{x y}$ and $k_{x y}$, called quadrature errors, are coupled damping and spring terms, respectively, mainly due to the asymmetries in suspension structure and misalignment of sensors and actuators. Bounded unknown disturbances are denoted by $d_{x}$ and $d_{y}$. It is noted that the coupled damping coefficient between $x$ - and $y$-axis is far smaller than $x$-axis or $y$-axis own damping coefficient, that is $d_{x y} \ll d_{x x}$ and $d_{x y} \ll d_{y y}$.

Rewriting the gyroscope dynamics into vector forms results in

$$
M \ddot{\mathbf{q}}+D \dot{\mathbf{q}}+K \mathbf{q}=\boldsymbol{\tau}-2 \Omega \dot{\mathbf{q}}+\boldsymbol{\tau}_{d},
$$

where $\mathbf{q}=\left[\begin{array}{l}x \\ y\end{array}\right], \boldsymbol{\tau}=\left[\begin{array}{l}u_{x} \\ u_{y}\end{array}\right], \boldsymbol{\tau}_{d}=\left[\begin{array}{l}d_{x} \\ d_{y}\end{array}\right], M=\left[\begin{array}{cc}m & 0 \\ 0 & m\end{array}\right], D=$ $\left[\begin{array}{ll}d_{x x} & d_{x y} \\ d_{x y} & d_{y y}\end{array}\right], K=\left[\begin{array}{ll}k_{x x} & k_{x y} \\ k_{x y} & k_{y y}\end{array}\right]$, and $\Omega=\left[\begin{array}{cc}0 & -m \Omega_{z} \\ m \Omega_{z} & 0\end{array}\right]$.

\section{Problem Formulation}

All the MEMS gyroscope parameters in (6) cannot be known precisely. This may arise because the MEMS gyroscope is being subjected to unknown angular rate or because the exact evaluation of the gyroscope's dynamic is too costly. The dynamical model contains model uncertainties, external disturbance, and even some nonlinearities. We assume the external disturbances are bounded; that is $\left\|\boldsymbol{\tau}_{d}\right\| \leq b_{d}$, for some unknown positive constant $b_{d}$.

Let $\mathbf{q}_{d}=\left[x_{d}, y_{d}\right]^{T}$ denote the desired trajectory of the proof mass in the $x-y$ plan.

Define the tracking error as follows:

$$
\mathbf{e}(t)=\mathbf{q}_{d}(t)-\mathbf{q}(t) .
$$

The sliding mode is defined as

$$
\mathbf{r}(t)=\dot{\mathbf{e}}(t)+\Lambda \mathbf{e}(t),
$$

where $\Lambda=\Lambda^{T}>0$ is a design parameter matrix. Differentiating $\mathbf{r}(t)$ and using (6), the MEMS gyroscope dynamics can be expressed as

$$
M \dot{\mathbf{r}}=-D \mathbf{r}-\boldsymbol{\tau}+\mathbf{f}(\mathbf{x})-\boldsymbol{\tau}_{d},
$$

where the unknown gyroscope function is

$$
\mathbf{f}(\mathbf{x})=M\left(\ddot{\mathbf{q}}_{d}+\Lambda \dot{\mathbf{e}}\right)+D\left(\dot{\mathbf{q}}_{d}+\Lambda \mathbf{e}\right)+K \mathbf{q}+2 \Omega \dot{\mathbf{q}},
$$

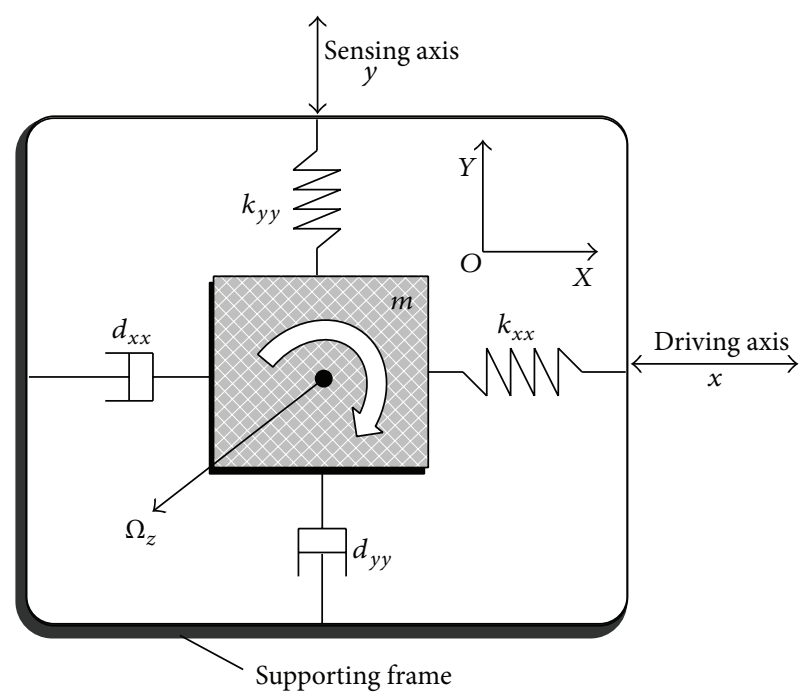

FIgURE 1: Schematic model of a two-axis MEMS vibratory gyroscope.

and $\mathbf{x} \in \mathfrak{R}^{10}$ are the available measured signals:

$$
\mathbf{x}=\left[\begin{array}{lllll}
\mathbf{e}^{T} & \dot{\mathbf{e}}^{T} & \mathbf{q}_{d}^{T} & \dot{\mathbf{q}}_{d}^{T} & \ddot{\mathbf{q}}_{d}^{T}
\end{array}\right]^{T} .
$$

If we can reconstruct an estimate of $\mathbf{f}(\mathbf{x})$ using $\mathbf{x}$ by some means not yet disclosed, we now define a control input as

$$
\boldsymbol{\tau}=\widehat{\mathbf{f}}(\mathbf{x})+K_{v} \mathbf{r},
$$

where gain matrix $K_{v}=K_{v}^{T}>0$ and $\widehat{\mathbf{f}}(\mathbf{x})$ is the estimate of $\mathbf{f}(\mathbf{x})$. The closed-loop system becomes

$$
\begin{aligned}
M \dot{\mathbf{r}} & =-D \mathbf{r}-\widehat{\mathbf{f}}-K_{v} \mathbf{r}+\mathbf{f}-\boldsymbol{\tau}_{d} \\
& =-\left(K_{v}+D\right) \mathbf{r}+\widetilde{\mathbf{f}}-\boldsymbol{\tau}_{d},
\end{aligned}
$$

where the functional estimation error is given by

$$
\widetilde{\mathbf{f}}=\mathbf{f}-\widehat{\mathbf{f}} .
$$

Equation (13) is an error system wherein the sliding mode is driven by the functional estimation error. The control $\boldsymbol{\tau}_{0}$ incorporates a proportional-plus-derivative (PD) term in $K_{v} \mathbf{r}=K_{v}(\dot{\mathbf{e}}+\Lambda \mathbf{e})$. In the next section we will use (13) to focus on selecting $\mathrm{NN}$ tuning algorithms that guarantee the stability of the sliding mode $\mathbf{r}(t)$.

\section{Adaptive Neural Network Controller}

4.1. RBF Neural Network. RBF neural network has the universal approximation property that states that any sufficiently smooth function can be approximated by a suitable large network for all inputs in a compact set and the resulting function reconstruction error is bounded. The RBF network has a fast convergence property and a simple architecture. Therefore, in this section we apply RBF network for compensating for the unknown dynamics part in Section 2. 


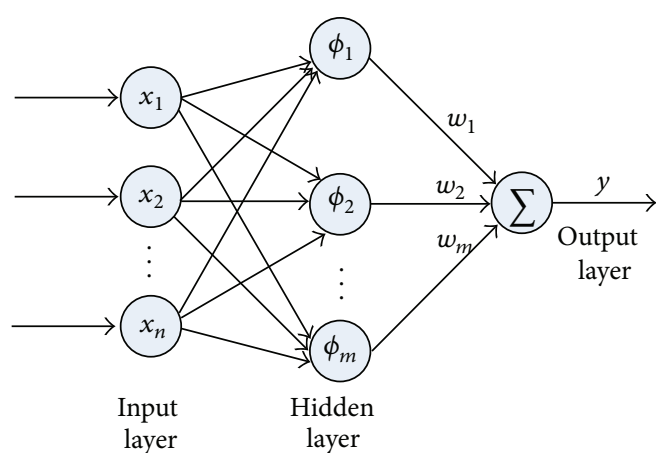

FIGURE 2: Architecture of RBF network.

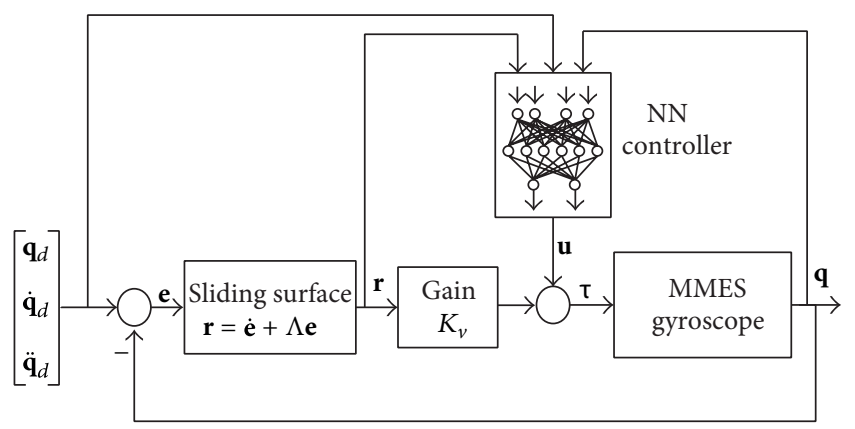

FIgURE 3: Adaptive NN control structure.

The structure of RBF neural network is a three-layer feed forward network shown as in Figure 2. The output of an RBF neural network can be described as

$$
\begin{gathered}
y_{i}=\sum_{j=1}^{n_{2}} \omega_{i j} \phi_{j}, \quad i=1,2, \ldots n_{3}, \\
\phi_{j}(\mathbf{x})=g\left(\frac{\left\|\mathbf{x}-\mathbf{c}_{j}\right\|}{\sigma_{j}}\right), \quad j=1,2, \ldots n_{2},
\end{gathered}
$$

where $n_{2}$ and $n_{3}$ are the numbers of hidden and output nodes, respectively, and $\mathbf{x} \in \mathfrak{R}^{n_{1}}$ and $\omega_{i j}$ is the weight connecting the $j$ th hidden node to the $i$ th output node. $y_{i}$ is the output of the $i$ th node. $\phi_{j}(\mathbf{x})$ is the $j$ th radial basis function. $\mathbf{c}_{j} \in \mathfrak{R}^{n_{1}}$ is the center vector and $\sigma_{j}$ represents the width of the $j$ th hidden node. The activation function $g$ is often chosen to be Gaussian function $g(\alpha)=\exp \left(-\alpha^{2}\right)$.

If the RBF net parameters $\mathbf{c}_{j}$ and $\boldsymbol{\sigma}_{j}$ are kept fixed, the only adjustable weights $\omega$ appear linearly with respect to the known nonlinearity $\phi(\mathbf{x})$. Now, the output of RBF NN can be rewritten as

$$
\mathbf{y}=W^{T} \boldsymbol{\phi}(\mathbf{x}),
$$

where $W^{T}=\left[\omega_{i j}\right]$ and $\phi(\mathbf{x})=\left[\phi_{j}(\mathbf{x})\right]$.

$\omega_{i j}$ is the weight connecting the $j$ th hidden node to the $i$ th output node. $\phi_{j}(\mathbf{x})$ is the $j$ th radial basis function.

Following the above results, the unknown MEMS gyroscope function $\mathbf{f}(\mathbf{x})$ can be parameterized by a RBF network with output $\widehat{\mathbf{f}}(\mathbf{x}, \widehat{W})$, where $\widehat{W}$ is the matrix of adjustable

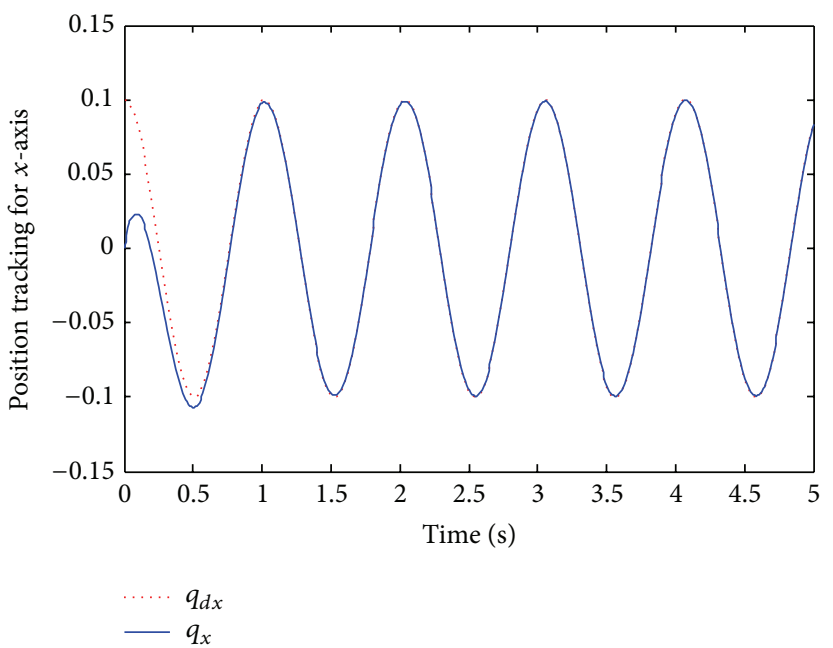

FIGURE 4: Position tracking of $x$-axis with adaptive neural sliding compensator.

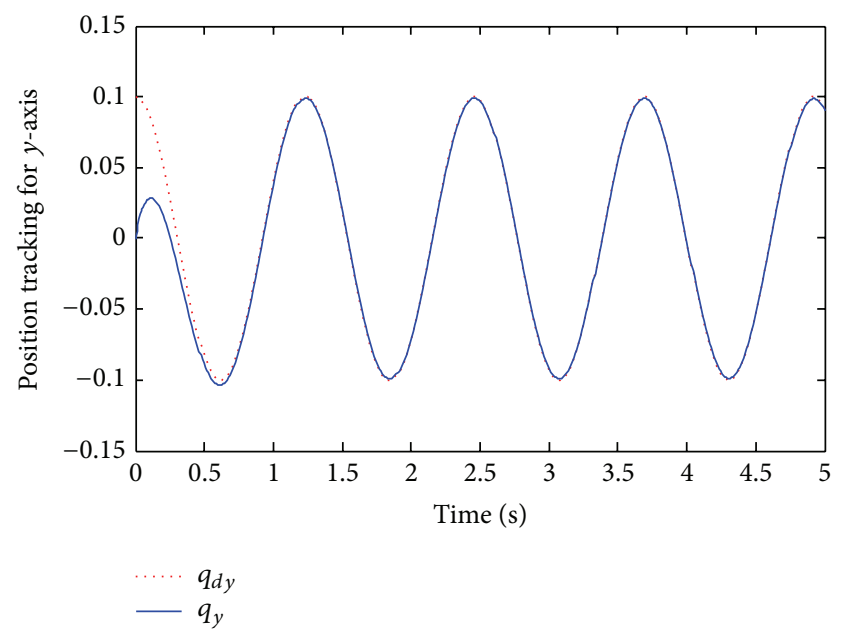

FIGURE 5: Position tracking of $y$-axis with adaptive neural sliding compensator.

weights. We assume that the function $\mathbf{f}(\mathbf{x})$ is given by a RBF NN for some constant "ideal" NN weights $W$, and the net reconstruction error $\boldsymbol{\varepsilon}(\mathbf{x})$ is bounded by an unknown constant $\varepsilon_{N}$ as

$$
\mathbf{f}(\mathbf{x})=W^{T} \phi(\mathbf{x})+\boldsymbol{\varepsilon}(\mathbf{x}),
$$

where $\boldsymbol{\varepsilon}(\mathbf{x})$, as mentioned above, is the NN functional reconstruction error vector. For the ideal NN weights, $\|\boldsymbol{\varepsilon}(\mathbf{x})\| \leq \varepsilon_{N}$.

Meanwhile, we assume that the ideal weights are bounded by

$$
\|W\| \leq W_{B} .
$$

4.2. Controller Structure and Error System Dynamics. Define the NN functional estimate of (10) as

$$
\widehat{\mathbf{f}}(\mathbf{x})=\widehat{W}^{T} \boldsymbol{\phi}(\mathbf{x})
$$




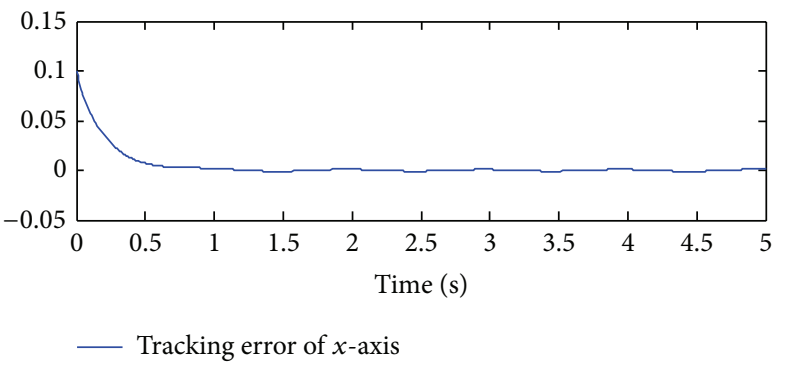

(a)

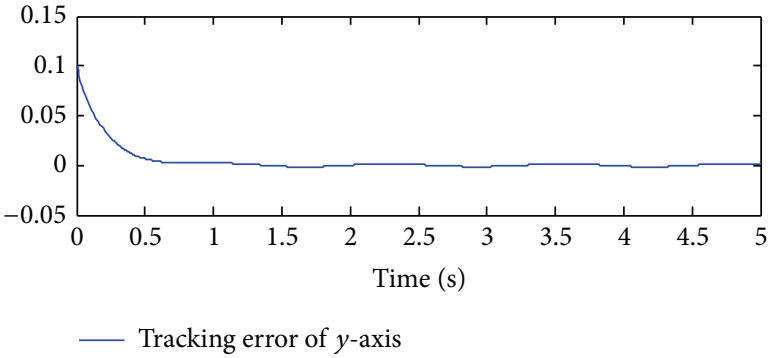

(b)

FIgURE 6: Tracking errors with adaptive neural sliding compensator.

with $\widehat{W}$ as the estimated values of the ideal RBF NN weights $W$.

Substituting (19) into (12) yields

$$
\boldsymbol{\tau}=W^{T} \phi(\mathbf{x})+K_{v} \mathbf{r} .
$$

The proposed NN control structure is shown in Figure 3.

Using this controller, the sliding mode dynamics become

$$
\begin{aligned}
M \dot{\mathbf{r}} & =-\left(K_{v}+D\right) \mathbf{r}+\widetilde{W}^{T} \boldsymbol{\phi}(\mathbf{x})+\left(\boldsymbol{\varepsilon}-\boldsymbol{\tau}_{d}\right) \\
& =-\left(K_{v}+D\right) \mathbf{r}+\boldsymbol{\varsigma}_{1},
\end{aligned}
$$

where $\widetilde{W}=W-\widehat{W}$ and $\boldsymbol{\varsigma}_{1}=\widetilde{w}^{T} \phi(\mathbf{x})+\left(\boldsymbol{\varepsilon}-\boldsymbol{\tau}_{d}\right)$.

Theorem 1. Let the designed trajectory be bounded, the control input for (6) given by (20), and weights tuning provided by

$$
\dot{\widehat{W}}=F \phi(\mathbf{x}) \mathbf{r}^{T}-\gamma F\|\mathbf{r}\| \widehat{W}
$$

with any constant gain matrix $F=F^{T}>0$ and scalar design parameter $\gamma>0$. Then, for large enough control gain $K_{v}$, the sliding mode $\mathbf{r}(t)$ and NN weight estimates $\widehat{W}$ are uniformly ultimate bounded. Moreover, the tracking error may be kept as small as desired by increasing the gains $K_{v}$ in (20).

Proof. Consider the following Lyapunov function candidate:

$$
V=\frac{1}{2} \mathbf{r}^{T} M \mathbf{r}+\frac{1}{2} \operatorname{tr}\left(\widetilde{W}^{\mathrm{T}} F^{-1} \widetilde{W}\right)
$$

where $\operatorname{tr}(\cdot)$ denotes the matrix trace operator; matrix $F$ is the adaptive law gain.

Differentiating (23) yields

$$
\begin{aligned}
\dot{V}= & \mathbf{r}^{T} M \dot{\mathbf{r}}+\operatorname{tr}\left(\widetilde{W}^{\mathrm{T}} F^{-1} \dot{\widetilde{W}}\right) \\
= & -\mathbf{r}^{T}\left(K_{v}+D\right) \mathbf{r}+\operatorname{tr}\left(\widetilde{W}^{T}\left(F^{-1} \dot{\widetilde{W}}+\phi(\mathbf{x}) \mathbf{r}^{T}\right)\right) \\
& +\mathbf{r}^{T}\left(\boldsymbol{\varepsilon}-\boldsymbol{\tau}_{d}\right) .
\end{aligned}
$$

Substituting (22) in (24) and using the fact that $\dot{\widetilde{W}}=-\dot{\widehat{W}}$ yield the following expression:

$\dot{V}=-\mathbf{r}^{T}\left(K_{v}+D\right) \mathbf{r}+\gamma\|\mathbf{r}\| \operatorname{tr}\left\{\widetilde{W}^{T}(W-\widetilde{W})\right\}+\mathbf{r}^{T}\left(\boldsymbol{\varepsilon}-\boldsymbol{\tau}_{d}\right)$.
Since $\operatorname{tr}\left\{\widetilde{W}^{\mathrm{T}}(W-\widetilde{W})\right\}=\langle\widetilde{W}, W\rangle_{F}-\|\widetilde{W}\|_{F}^{2} \leq\|\widetilde{W}\|_{F}\|W\|_{F}-$ $\|\widetilde{W}\|_{F}^{2},(25)$ yields

$$
\begin{aligned}
\dot{V} \leq & -\mathbf{r}^{T} K_{v} \mathbf{r}+\gamma\|\mathbf{r}\| \cdot\|\widetilde{W}\|_{F}\left(\|W\|_{F}-\|\widetilde{W}\|_{F}\right) \\
& +\left(\varepsilon_{N}+b_{d}\right)\|\mathbf{r}\| \\
\leq & -K_{v \min }\|\mathbf{r}\|^{2}+\gamma\|\mathbf{r}\| \cdot\|\widetilde{W}\|_{F}\left(W_{B}-\|\widetilde{W}\|_{F}\right) \\
& +\left(\varepsilon_{N}+b_{d}\right)\|\mathbf{r}\| \\
= & -\|\mathbf{r}\|\left[K_{v \min }\|\mathbf{r}\|+\gamma\|\widetilde{W}\|_{F}\right. \\
& \left.\times\left(\|\widetilde{W}\|_{F}-W_{B}\right)-\left(\varepsilon_{N}+b_{d}\right)\right],
\end{aligned}
$$

which is negative as long as the term in braces is positive. Completing the square yields

$$
\begin{aligned}
K_{v \min } & \|\mathbf{r}\|+\gamma\|\widetilde{W}\|_{F}\left(\|\widetilde{W}\|_{F}-W_{B}\right)-\left(\varepsilon_{N}+b_{d}\right) \\
& =\gamma\left(\|\widetilde{W}\|_{F}-\frac{W_{B}}{2}\right)^{2}-\frac{\gamma W_{B}^{2}}{4}+K_{v \min }\|\mathbf{r}\|-\left(\varepsilon_{N}+b_{d}\right),
\end{aligned}
$$

which is guaranteed positive as long as

$$
\|\mathbf{r}\|>\frac{\gamma W_{B}^{2} / 4+\left(\varepsilon_{N}+b_{d}\right)}{K_{v \min }} \equiv b_{r}
$$

or

$$
\|\widetilde{W}\|_{F}>\frac{W_{B}}{2}+\sqrt{\frac{\gamma W_{B}^{2}}{4}+\frac{\varepsilon_{N}+b_{d}}{\gamma}} \equiv b_{W} .
$$

Thus, $\dot{V}$ is negative outside a compact set; this demonstrates the UUB (uniformly ultimate bounded) of both $\|\mathbf{r}\|$ and $\|\widetilde{W}\|_{F}$. Since any excursions of $\|\mathbf{r}\|$ or $\|\widetilde{W}\|_{F}$ beyond the bounds given in (28) and (29), respectively, lead to a decrease in the Lyapunov function $L$, it follows that the right-hand sides of (28) and (29) can be taken as practical bounds on $\|\mathbf{r}\|$ and $\|\widetilde{W}\|_{F}$, respectively. Note from (28), moreover, that arbitrarily small error bounds may be achieved by selecting large tracking control gains $K_{v}$. 


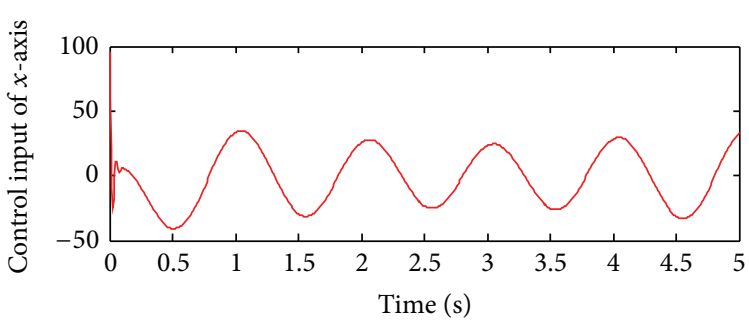

(a)

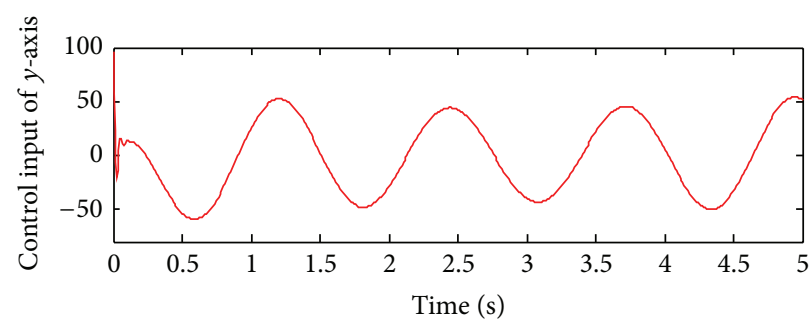

(b)

FIGURE 7: Control inputs for gyroscope with adaptive neural sliding compensator $\left((\mathrm{a}): u_{x} ;(\mathrm{b}): u_{y}\right)$.

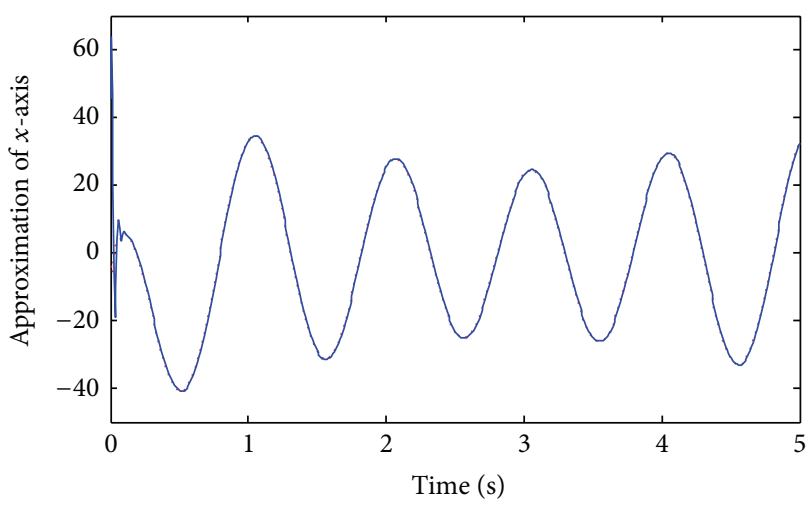

..... Actual

— Estimation

FIGURE 8: RBF network approximation of $x$-axis component with adaptive neural sliding compensator.

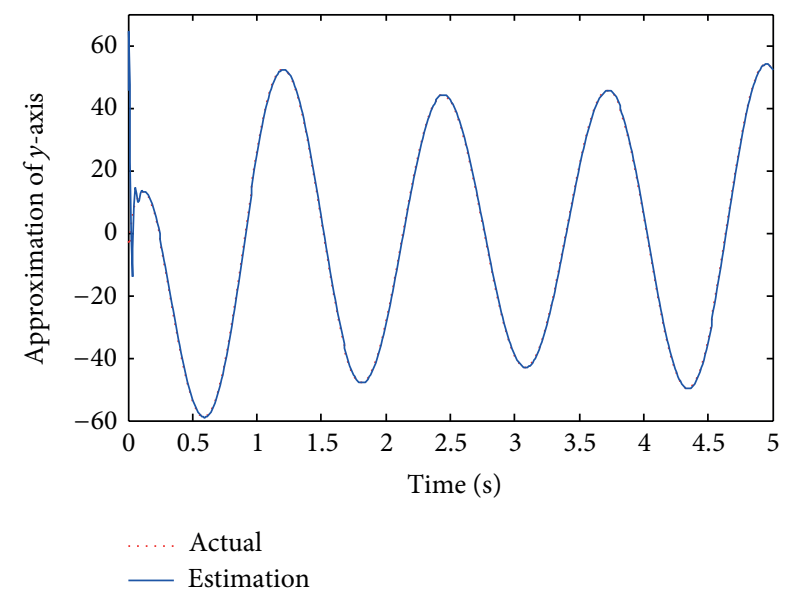

FIGURE 9: RBF network approximation of $y$-axis component with adaptive neural sliding compensator.

Remark 2. Given $A=\left[a_{i j}\right]$, the Frobenius norm is defined by

$$
\|A\|_{F}^{2}=\operatorname{tr}\left(A^{T} A\right)=\sum a_{i j}^{2} .
$$

The associated inner product is $\langle A, B\rangle_{F}=\operatorname{tr}\left(A^{T} B\right)$, where the dimensions of $A$ and $B$ are suitable for matrix product.

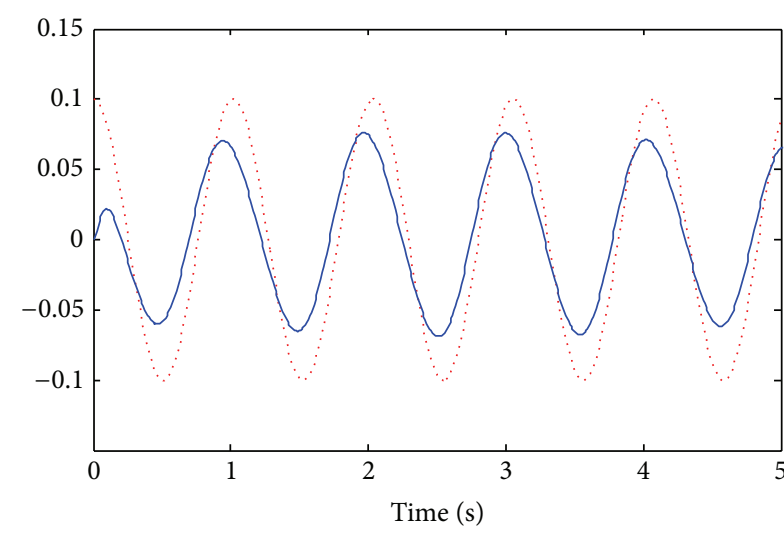

$\cdots q_{d x}$

FIGURE 10: Position tracking of $x$-axis without NN controller.

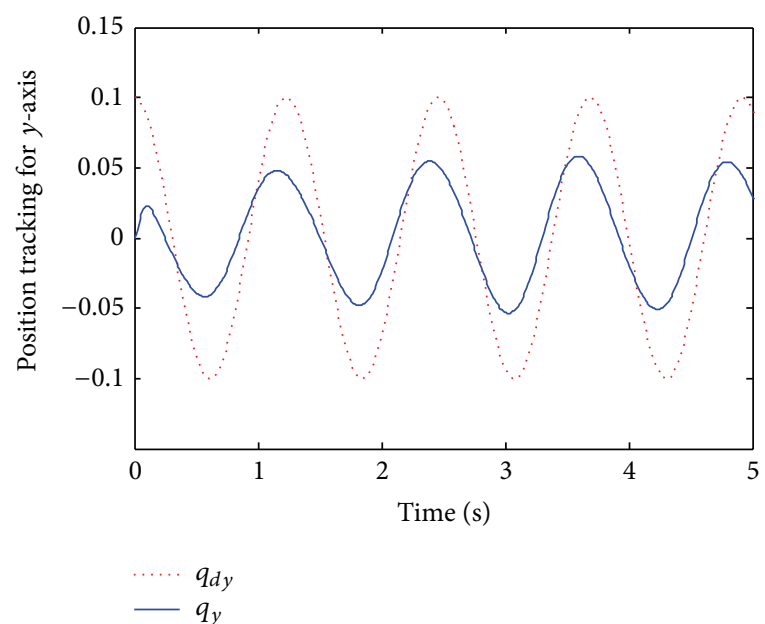

FIGURE 11: Position tracking of $y$-axis without NN controller.

\section{Simulation Analysis}

In this section, we will evaluate the proposed adaptive neural network scheme, which will require no knowledge of the dynamics, not even their structure on the lumped MEMS gyroscope sensor model $[3,4]$. The control objective is to maintain the gyroscope system to track the desired reference 


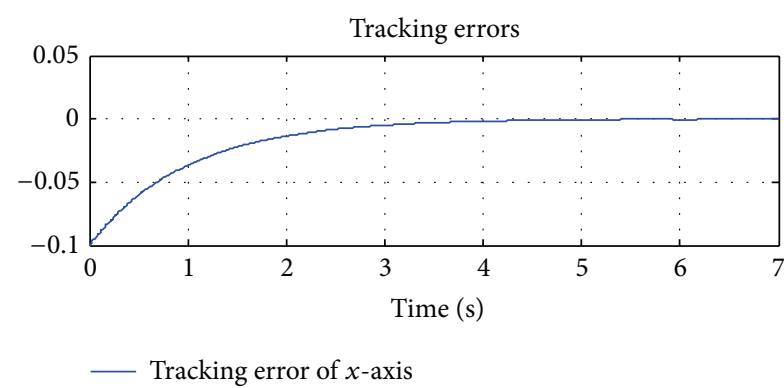

(a)

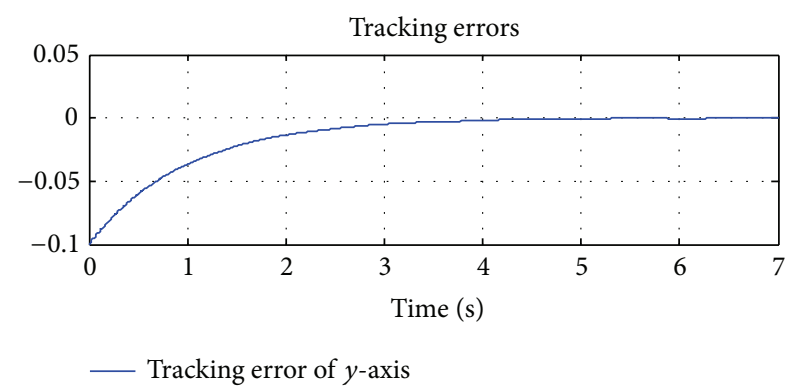

(b)

FIGURE 12: Tracking errors with neural compensator.

trajectory and estimate the unknown function $\mathbf{f}(\mathbf{x})$ as in (10). The parameters of the MEMS gyroscope are shown as

$$
\begin{gathered}
m=1.8 \times 10^{-7} \mathrm{~kg}, \quad k_{x x}=63.955 \mathrm{~N} / \mathrm{m}, \\
k_{y y}=95.92 \mathrm{~N} / \mathrm{m}, \quad k_{x y}=12.779 \mathrm{~N} / \mathrm{m}, \\
d_{x x}=1.8 \times 10^{-6} \mathrm{~N} \cdot \mathrm{s} / \mathrm{m}, \quad d_{y y}=1.8 \times 10^{-6} \mathrm{~N} \cdot \mathrm{s} / \mathrm{m}, \\
d_{x y}=3.6 \times 10^{-7} \mathrm{~N} \cdot \mathrm{s} / \mathrm{m} .
\end{gathered}
$$

The unknown angular velocity is assumed $\Omega_{z}=100 \mathrm{rad} / \mathrm{s}$. The desired trajectory is defined as $q_{d x}=0.1 * \cos \left(\omega_{1} t\right), q_{d y}=$ $0.1 * \cos \left(\omega_{2} t\right)$, where $\omega_{1}=6.17$ and $\omega_{2}=5.11$.

The external disturbance is $\tau_{d}=\left[(\sin (6.17 * t))^{2}+\right.$ $\left.\cos (6.17 * t),(\sin (5.11 * t))^{2}+\cos (5.11 * t)\right]^{T}$.

The gain matrix $K_{v}$ was taken as $K_{v}=\operatorname{diag}\{50,50\}$, sliding parameter $\Lambda=\operatorname{diag}\{5,5\}$. The RBF neural network is composed of 45 nodes. As we will see from the subsequent figure that the unique PD controller $\left(K_{v} \mathbf{r}\right)$ could ensure that the tracking error belongs to a compact set. Thus, when we design the RBF net centroids, we constrain our attention on that compact set and take width $\sigma=1$. The response with these controller parameters when $\mathbf{q}=\mathbf{0}$ and $\dot{\mathbf{q}}=\mathbf{0}$ is shown in the following figures.

Figures 4 and 5 show the proof mass along $x$-axis and $y$-axis tracking trajectories, respectively. Figure 6 depicts the tracking errors. It can be seen that the tracking error belongs to a small region with the adaptive RBF sliding compensator. The control inputs appear in Figure 7. As seen from the figure, the control inputs are continuous and smooth with respect to time, which implies that the proposed controller is easy to implement. Figures 8 and 9 show the approximation of RBF network to unknown MEMS gyroscope function $\mathbf{f}(\mathbf{x})$. The approximation result is impressive because of the satisfactory performance of the RBF NN. As shown in the two figures, RBF NN could approximate the unknown gyroscope function quickly using the available measured signals $\mathbf{x}$ throughout the whole compact set.

In order to demonstrate the contribution of RBF NN, the response without RBF NN is also discussed and shown as in Figures 10 and 11. The responses with the controller $\boldsymbol{\tau}=K_{v} \mathbf{r}$, that is, with no neural network, are drawn. A PD controller should give bounded errors if $K_{v}$ is large enough and the

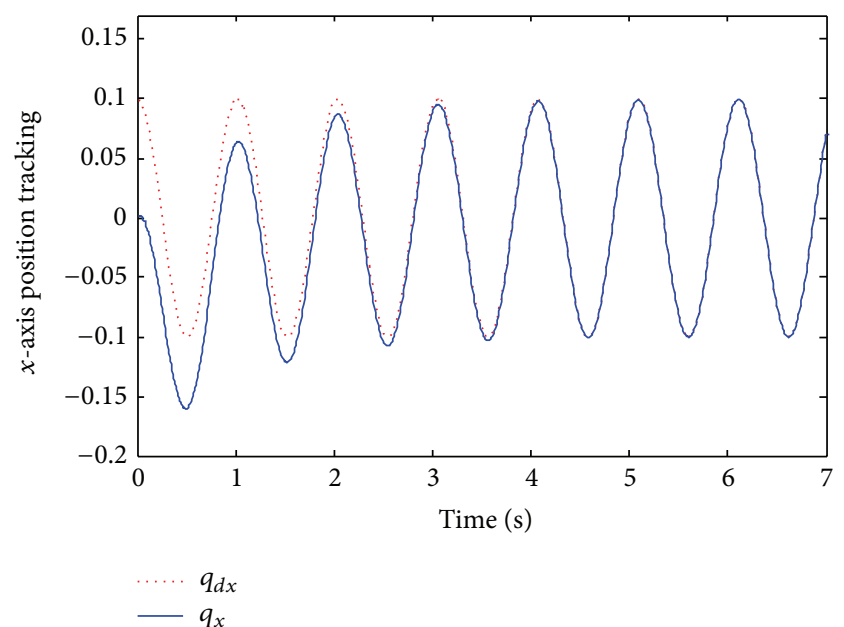

FIGURE 13: $x$-axis position tracking with neural compensator.

tracking errors belong to a compact set. It is very clear that the addition of the NN makes a very significant improvement in the tracking performance.

To compare the performance of the proposed controller and the neural compensator without sliding mode method in [16], Figures 12, 13, and 14 show the response with the neural compensator under the same gyroscope parameters and disturbances introduced herein.

The tracking results with neural compensator show clearly that both the convergence time and the ultimate tracking error bound are worse than the proposed neural sliding mode controller, demonstrating that adaptive sliding mode controller using neural network approximation could improve the dynamic and static performance.

\section{Conclusion}

The trajectory control of the MEMS gyroscope using adaptive NN controller is investigated. A novel weight tuning algorithm designed based on Lyapunov stability theorem is derived to establish the bounded tracking errors and NN weights, which consists of a standard term plus robust signal that provides the possibility of arbitrary small tracking error. It is clearly observed that the MEMS gyroscope function 


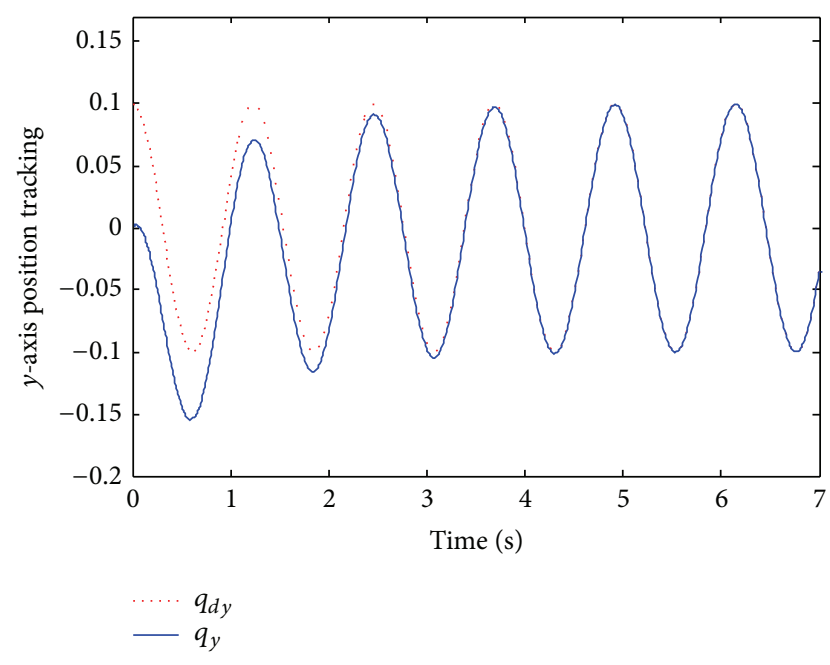

FIGURE 14: $y$-axis position tracking with neural compensator.

$\mathbf{f}(\mathbf{x})$ can be effectively approximated by its estimate $\widehat{\mathbf{f}}(\mathbf{x})$ using the RBF neural network and the tracking error of the proof mass can be greatly improved in the presence of model uncertainties and external disturbances. Numerical simulations verify the effectiveness of the proposed adaptive NN compensation scheme to cope with all structured and unstructured uncertainties existing in the MEMS gyroscope.

\section{Conflict of Interests}

The authors declare that there is no conflict of interests regarding the publication of this paper.

\section{Acknowledgments}

The authors thank the anonymous reviewers for their useful comments that improved the quality of the paper. This work is partially supported by National Science Foundation of China under Grant no. 61374100, Natural Science Foundation of Jiangsu Province under Grant no. BK20131136, the University Graduate Research and Innovation Projects of Jiangsu Province under Grant no. CXLX12_0235, and the Fundamental Research Funds for the Central Universities under Grant no. 2013B24614.

\section{References}

[1] R. P. Leland, "Adaptive control of a MEMS gyroscope using lyapunov methods," IEEE Transactions on Control Systems Technology, vol. 14, no. 2, pp. 278-283, 2006.

[2] S. Park, R. Horowitz, S. K. Hong, and Y. Nam, "Trajectoryswitching algorithm for a MEMS gyroscope," IEEE Transactions on Instrumentation and Measurement, vol. 56, no. 6, pp. 25612569, 2007.

[3] C. Batur, T. Sreeramreddy, and Q. Khasawneh, "Sliding mode control of a simulated MEMS gyroscope," ISA Transactions, vol. 45, no. 1, pp. 99-108, 2006.
[4] J. Fei and C. Batur, "A novel adaptive sliding mode control with application to MEMS gyroscope," ISA Transactions, vol. 48, no. 1, pp. 73-78, 2009.

[5] N.-C. Tsai and C.-Y. Sue, "Integrated model reference adaptive control and time-varying angular rate estimation for micromachined gyroscopes," International Journal of Control, vol. 83, no. 2, pp. 246-256, 2010.

[6] J. Raman, E. Cretu, P. Rombouts, and L. Weyten, "A closedloop digitally controlled MEMS gyroscope with unconstrained sigma-delta force-feedback," IEEE Sensors Journal, vol. 9, no. 3, pp. 297-305, 2009.

[7] F. Lewis, S. Jagannathan, and A. Yesildirek, Neural Network Control of Robot Manipulators, Taylor and Francis, 1999.

[8] F. L. Lewis, K. Liu, and A. Yesildirek, "Neural net robot controller with guaranteed tracking performance," IEEE Transactions on Neural Networks, vol. 6, no. 3, pp. 703-715, 1995.

[9] G. Feng, "A compensating scheme for robot tracking based on neural networks," Robotics and Autonomous Systems, vol. 15, no. 3, pp. 199-206, 1995.

[10] S.-C. Lin and Y.-Y. Chen, "RBF network-based sliding mode control," in Proceedings of the IEEE International Conference on Systems, Man and Cybernetics, pp. 1957-1961, October 1994.

[11] B. S. Park, S. J. Yoo, J. B. Park, and Y. H. Choi, "Adaptive neural sliding mode control of nonholonomic wheeled mobile robots with model uncertainty," IEEE Transactions on Control Systems Technology, vol. 17, no. 1, pp. 207-214, 2009.

[12] N. Sadati and R. Ghadami, "Adaptive multi-model sliding mode control of robotic manipulators using soft computing," Neurocomputing, vol. 71, no. 13-15, pp. 2702-2710, 2008.

[13] M.-J. Lee and Y.-K. Choi, "An adaptive neurocontroller using RBFN for robot manipulators," IEEE Transactions on Industrial Electronics, vol. 51, no. 3, pp. 711-717, 2004.

[14] F.-J. Lin, S.-Y. Chen, and K.-K. Shyu, "Robust dynamic slidingmode control using adaptive RENN for magnetic levitation system," IEEE Transactions on Neural Networks, vol. 20, no. 6, pp. 938-951, 2009.

[15] S.-J. Huang, K.-S. Huang, and K.-C. Chiou, "Development and application of a novel radial basis function sliding mode controller," Mechatronics, vol. 13, no. 4, pp. 313-329, 2003.

[16] J. Fei and Y. Yang, "Adaptive neural compensation scheme for robust tracking of MEMS gyroscopes," in Proceedings of IEEE Conference on SMC, pp. 1546-1551, Seoul, Korea, 2012.

[17] J. Fei and M. Xin, "An adaptive fuzzy sliding mode controller for MEMS triaxial gyroscope with angular velocity estimation," Nonlinear Dynamics, vol. 24, no. 1, pp. 201-210, 2012.

[18] J. Fei and H. Ding, "Adaptive neural sliding mode control of MEMS triaxial gyroscope based on feedback linearization approach," in Proceedings of Annual Conference of SICE, pp. 1271-1276, Akita, Japan, 2012. 


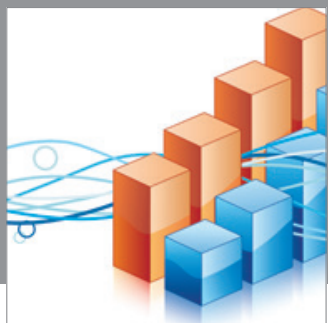

Advances in

Operations Research

mansans

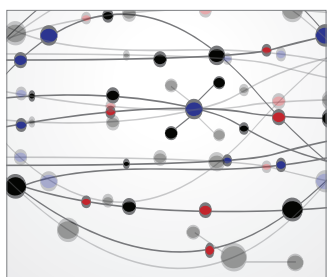

The Scientific World Journal
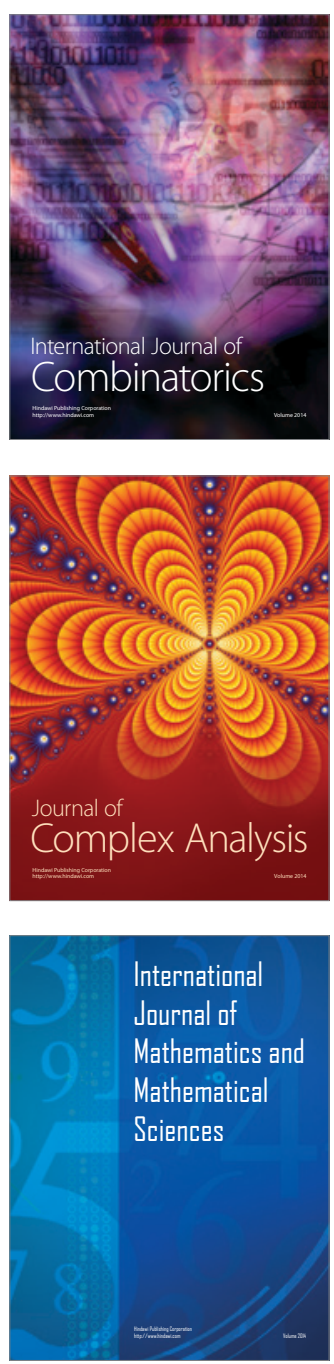
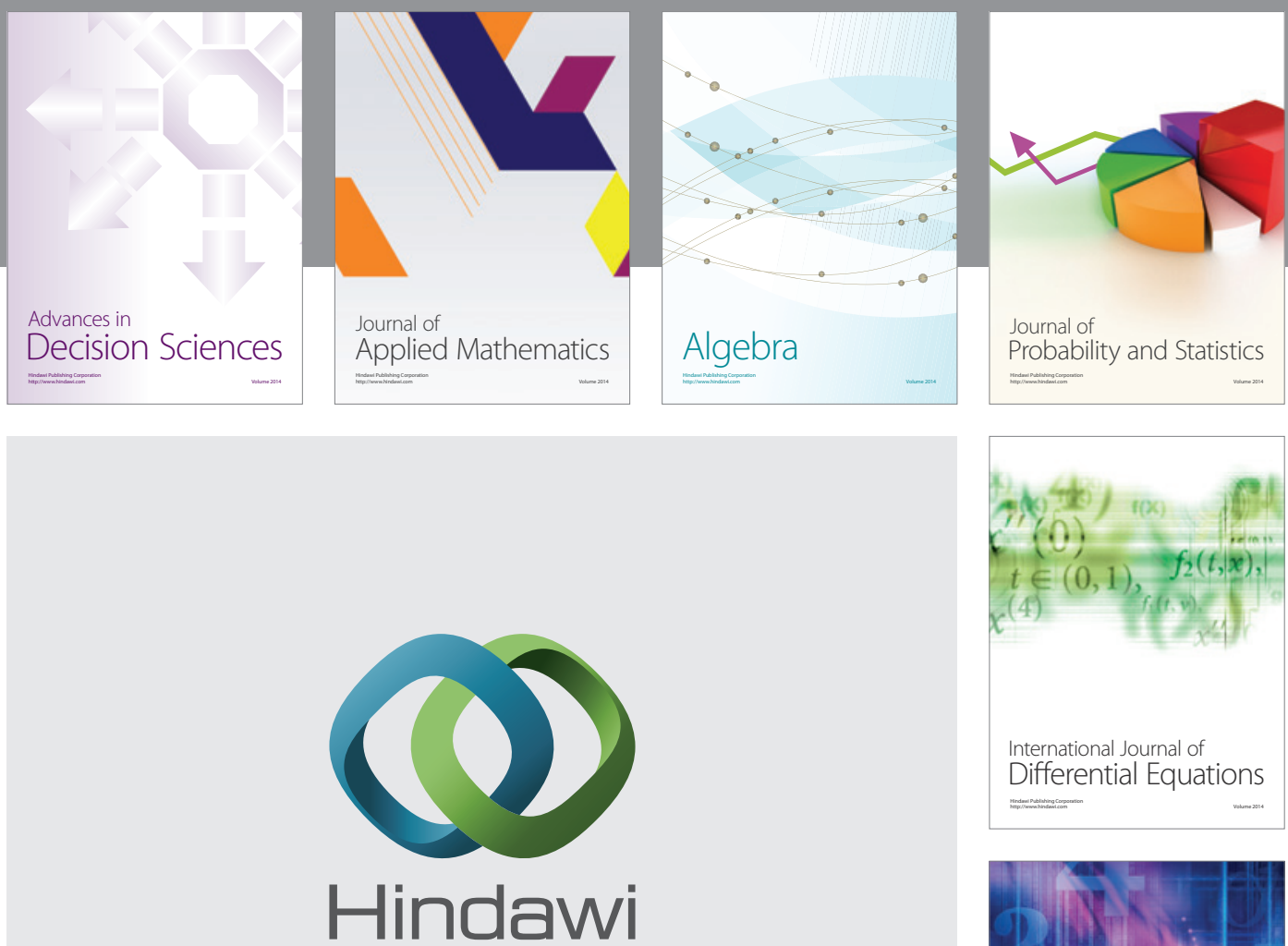

Submit your manuscripts at http://www.hindawi.com
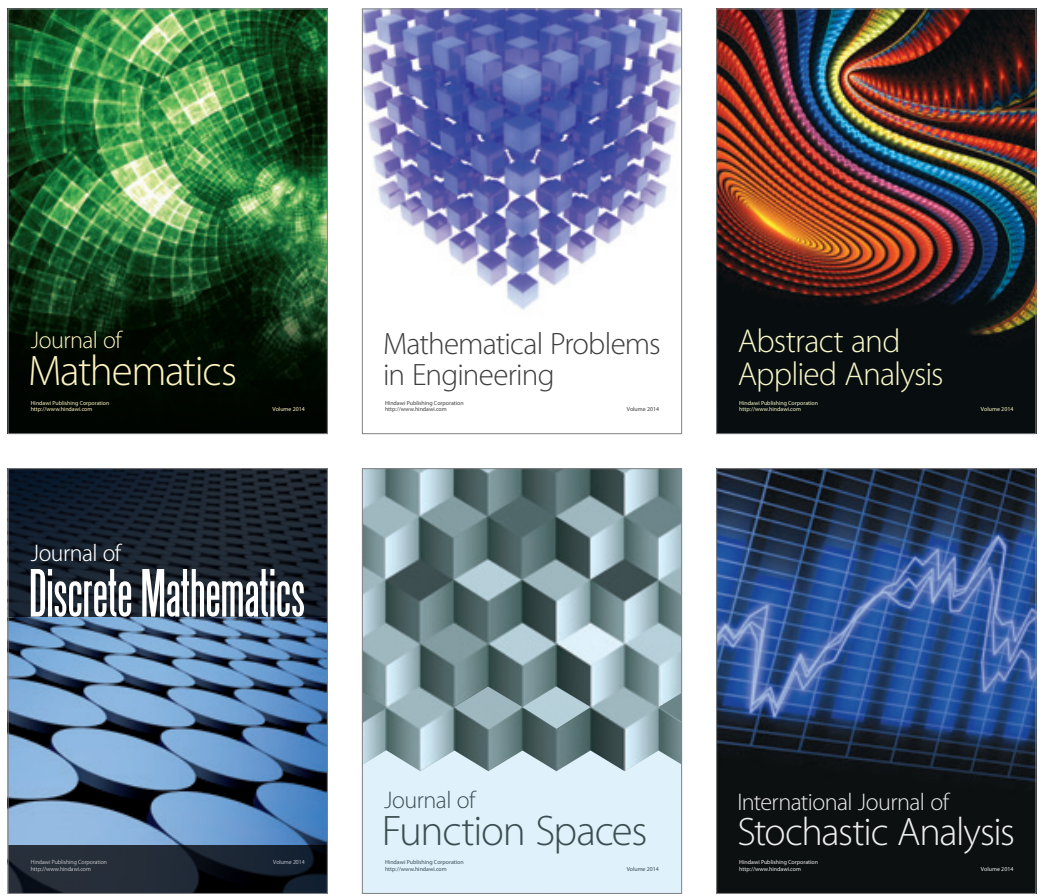

Journal of

Function Spaces

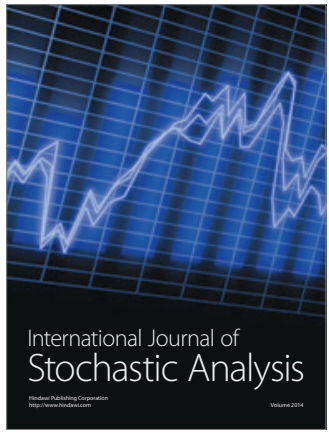

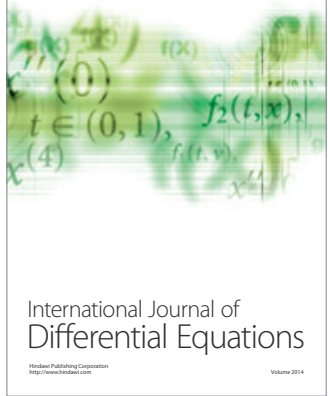
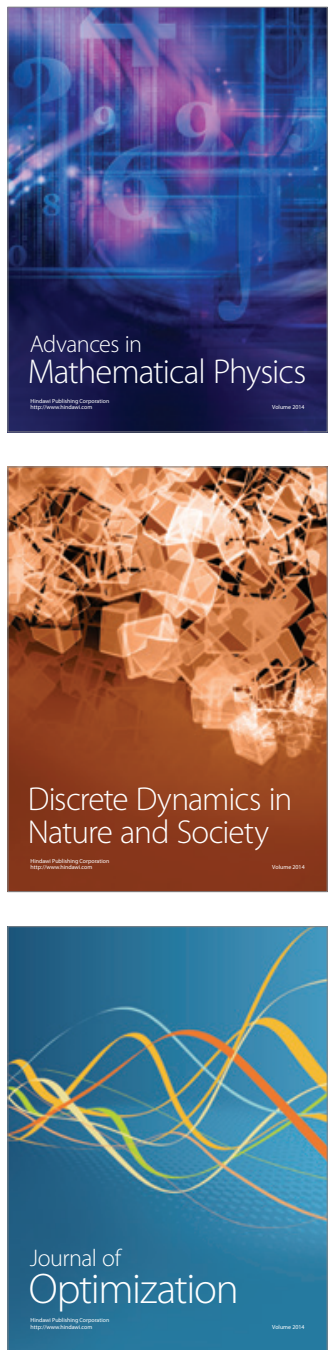